\title{
Derotation of the cosmic microwave background polarization: Full-sky formalism
}

\author{
Vera Gluscevic, ${ }^{1}$ Marc Kamionkowski, ${ }^{1}$ and Asantha Cooray ${ }^{2}$ \\ ${ }^{1}$ California Institute of Technology, Mail Code 350-17, Pasadena, California 91125, USA \\ ${ }^{2}$ Department of Physics and Astronomy, University of California, Irvine, California 92697, USA
}

(Received 12 May 2009; published 15 July 2009)

\begin{abstract}
Mechanisms have been proposed that might rotate the linear polarization of the cosmic microwave background $(\mathrm{CMB})$ as it propagates from the surface of last scatter. In the simplest scenario, the rotation will be uniform across the sky, but the rotation angle may also vary across the sky. We develop in detail the complete set of full-sky quadratic estimators for the rotation of the $\mathrm{CMB}$ polarization that can be constructed from the $\mathrm{CMB}$ temperature and polarization. We derive the variance with which these estimators can be measured and show that these variances reduce to the simpler flat-sky expressions in the appropriate limit. We evaluate the variances numerically. While the flat-sky formalism may be suitable if the rotation angle arises as a realization of a random field, the full-sky formalism will be required to search for rotations that vary slowly across the sky as well as for models in which the angular power spectrum for the rotation angle peaks at large angles.
\end{abstract}

DOI: 10.1103/PhysRevD.80.023510

PACS numbers: 98.80.-k, 98.70.Vc, 98.80.Cq

\section{INTRODUCTION}

Great strides have been made during the past decade in obtaining precise maps of the cosmic microwave background (CMB) temperature and polarization [1]. Yet there are still increasingly precise data to come [2]. Inflation [3], which has passed a number of tests so far, will be tested further [4], and we may begin to understand the physics responsible for inflation. Moreover, there will be a number of different types of departures from the standard scenario that can be detected with new measurements.

The CMB polarization pattern can be decomposed into a gradient component ( $E$ modes) and a curl component $(B$ modes) $[5,6]$. A given inflationary model makes specific predictions for the power spectra of these components. For example, if the model predicts no primordial gravitational waves, then there is no $B$ mode at the CMB surface of last scatter. If there is a primordial gravitational-wave background with a sufficiently large amplitude, there will be $B$ modes at the surface of last scatter at a detectable level [7], and with a characteristic power spectrum.

Since the post-recombination Universe is transparent to the $\mathrm{CMB}$, it is natural to assume that the polarization pattern that we see is the polarization pattern at the surface of last scatter. However, exotic mechanisms may rotate the linear polarization as it propagates through the Universe. For example, if cosmic acceleration is due to a quintessence field $\phi$, and if that field couples to the pseudoscalar $\tilde{F} F$ of electromagnetism, then the time variation of $\phi$ leads to a rotation of the linear polarization by an angle $\alpha$ [8]. If $\phi$ is spatially homogeneous, then the rotation-angle $\alpha$ is uniform across the sky. In this case, there is a characteristic parity-violating EB correlation induced in the CMB polarization [9]. Null measurements of this effect constrain $\alpha$ to be smaller than a few degrees $[10,11]$.
It may well be, however, that $\phi$ has spatial variations, in which case the rotation-angle $\alpha(\hat{\mathbf{n}})$ will vary with position $\hat{\mathbf{n}}$ on the sky [12]. There may also be mechanisms involving coupling of photons to dark matter that cause a spatially-varying $\alpha(\hat{\mathbf{n}})$ [13]. In either case, the EB power spectrum would include the dependence on $\alpha(\hat{\mathbf{n}})$, which would differentiate this scenario from the uniform-rotation case. For example, the EB power spectrum even vanishes [12] if $\alpha$ has a dipolar variation across the sky, in which case the positive EB correlation on one half of the sky is cancelled by a negative EB correlation of equal magnitude on the other half.

Ref. [14] showed that the rotation-angle $\alpha(\hat{\mathbf{n}})$ can be reconstructed, as a function of position on the sky, from the measured polarization map. If primordial perturbations are assumed to be Gaussian (as predicted by inflation), then the rotation introduces a characteristic non-Gaussian signal in the polarization map. Measurement of this non-Gaussian signal then provides the rotation angle. More specifically, if primordial perturbations are Gaussian, then the sphericalharmonic coefficients $E_{l m}$ and $B_{l m}$ of the polarization map are statistically independent for different $l$ and $m$. However, the rotation introduces off-diagonal correlations, i.e. correlations between different $l \mathrm{~m}$ pairs.

Ref. [15] revisited this proposal. The authors worked out the off-diagonal correlations that would be induced in the flat-sky limit, and evaluated numerically how well the small-angle analogues of the spherical-harmonic coefficients $\alpha_{L M}$ of the rotation angle could be measured with future satellite experiments like Planck [16] and CMBPol [17].

In this paper, we work out in more detail the full-sky formalism of Ref. [14]. We write down the minimumvariance estimator $\hat{\alpha}_{L M}$ for the rotation-angle sphericalharmonic coefficients that can be obtained from a full-sky 
CMB temperature/polarization map. We derive expressions for the variance with which the $\alpha_{L M}$ can be determined and show that they recover, in the large- $L$ limit, the correct flat-sky expressions. While a flat-sky analysis may be suitable if the rotation-angle power spectrum peaks at small scales, the full-sky formalism will be required to maximize the sensitivity in models, such as that in Ref. [12], where the signal-to-noise peaks at low $L$ (see, e.g., Fig. 1 in Ref. [15]). There has also been growing attention recently to the possibility that there may be variations in fundamental fields over distance scales comparable to, or larger than, the horizon (perhaps remnants of the preinflationary Universe) [18]. Observationally, these entail searches for departures from homogeneity/isotropy or departures from statistical homogeneity/isotropy in the CMB or large-scale structure $[19,20]$. The full-sky formalism we present here can be used to search for the low- $L$ (e.g., $L=1,2,3, \cdots)$ ) moments of $\alpha(\hat{\mathbf{n}})$ that may arise if $\phi$ has long-wavelength fluctuations, in addition to, or instead of, the higher- $L$ modes that can also be probed with a survey of a smaller region of the sky. ${ }^{1}$

The plan of the paper is as follows. In Sec. II, we derive the temperature/polarization correlations induced by rotation. In Sec. III, we construct the complete set of quadratic estimators for the rotation of the CMB polarization that can be constructed from the $\mathrm{CMB}$ temperature and polarization maps. We also determine the variances with which these estimators can be measured with a full-sky CMB map. Sec. IV derives the flat-sky limit of our variance expressions and compares them with previous work. Sec. V presents numerical results for the variances. Concluding remarks are provided in Sec. VI. Appendix A provides some useful formulas. Appendix B shows how the effects of weak lensing and rotation can be distinguished geometrically. Appendix $\mathrm{C}$ shows that our variance expressions retrieve successfully the expressions for a uniform rotation angle. And Appendix D illustrates the relative contribution of different $l l^{\prime}$ pairs to the rotation-angle estimator.

\section{CORRELATIONS INDUCED BY ROTATION}

In this section, we derive the temperature/polarization correlations that are induced by a post-recombination rotation of the polarization.

\section{A. Induced modes}

We begin by recalling that the Stokes parameters $Q(\hat{\mathbf{n}})$ and $U(\hat{\mathbf{n}})$, as a function of position $\hat{\mathbf{n}}$ on the sky, are components of a symmetric trace-free $2 \times 2$ tensor, $^{2}$

\footnotetext{
${ }^{1}$ The full-sky formalism is exact; note, however, that it is not computationally more demanding than the flat-sky calculations.

${ }^{2}$ Note that the definition of $P_{a b}$ here differs from that in Refs. [5] so that the normalization of the power spectra agree with those of Refs. [6].
}

$$
P_{a b}(\hat{n})=\frac{1}{\sqrt{2}}\left(\begin{array}{cc}
Q(\hat{n}) & -U(\hat{n}) \sin \theta \\
-U(\hat{n}) \sin \theta & -Q(\hat{n}) \sin ^{2} \theta
\end{array}\right) .
$$

This tensor can be expanded in terms of tensor spherical harmonics $Y_{(l m) a b}^{E}(\hat{\mathbf{n}})$ and $Y_{(l m) a b}^{B}(\hat{\mathbf{n}})$ in the usual fashion $[5,6]$,

$$
P_{a b}(\hat{n})=\sum_{l=2}^{\infty} \sum_{m=-l}^{l}\left[E_{l m} Y_{(l m) a b}^{E}(\hat{n})+B_{l m} Y_{(l m) a b}^{B}(\hat{n})\right],
$$

where $E_{l m}$ are the $E$-mode tensor-spherical-harmonic coefficients, and $B_{l m}$ are the $B$-mode tensor-sphericalharmonic coefficients.

We now suppose that the polarization at the surface of last scatter is a pure $E$ mode; we comment on the validity of this assumption below. Then, a small rotation $\alpha(\hat{n})$ induces a change to the polarization,

$$
\delta P_{a b}(\hat{n})=2 \alpha(\hat{n}) P_{a b}^{r}(\hat{n})
$$

where [14]

$$
P_{a b}^{r}(\hat{n})=\sum_{l=2}^{\infty} \sum_{m=-l}^{l} E_{l m} Y_{(l m) a b}^{B} .
$$

A pure $E$ mode thus gets rotated into a $B$ mode. Note that Eq. (4) is valid only in the limit of small rotations, $\alpha(\hat{\mathbf{n}}) \ll$ 1 . Given that, the $B$ mode will be small compared to the $\mathrm{E}$ mode, which satisfies existing empirical constraints.

Rotation is a scalar field on the sky and can thus be expanded in terms of spherical harmonics,

$$
\alpha(\hat{n})=\sum_{L M} \alpha_{L M} Y_{L M}(\hat{n}),
$$

where $\alpha_{L M}$ are the coefficients of the expansion.

Using Eqs. (3)-(5), the $B$ mode induced by a small rotation angle from a pure $E$ mode can be expressed as

$$
\begin{aligned}
\delta B_{l m} & =\int d \hat{n} \delta P_{a b}(\hat{n}) Y_{(l m)}^{B * a b}(\hat{n}) \\
& =2 \sum_{L M} \sum_{l_{2} m_{2}} \alpha_{L M} E_{l_{2} m_{2}} \int d \hat{n} Y_{(l m)}^{B *, a b} Y_{(L M)} Y_{\left(l_{2} m_{2}\right) a b}^{B} .
\end{aligned}
$$

Similarly, the induced $E$ mode is

$$
\begin{aligned}
\delta E_{l m} & =\int d \hat{n} \delta P_{a b}(\hat{n}) Y_{(l m)}^{E *, a b}(\hat{n}) \\
& =2 \sum_{L M} \sum_{l_{2} m_{2}} \alpha_{L M} E_{l_{2} m_{2}} \int d \hat{n} Y_{(l m)}^{E *, a b} Y_{(L M)} Y_{\left(l_{2} m_{2}\right) a b}^{B}
\end{aligned}
$$

We further develop the last two expressions using [5]

$$
Y_{(l m) a b}^{B}=\frac{N_{l}}{2}\left(Y_{(l m): a c} \varepsilon_{b}^{c}+Y_{(l m): b c} \varepsilon_{a}^{c}\right),
$$




$$
Y_{(l m) a b}^{E}=N_{l}\left(Y_{(l m): a b}-\frac{1}{2} g_{a b} Y_{(l m): c}{ }^{c}\right),
$$

where $g$ and $\varepsilon$ are, respectively, the metric tensor and the Levi-Civita tensor on a unit 2-sphere (see Appendix A); a colon denotes a covariant derivative on the 2-sphere; and $N_{l}$ is given by

$$
N_{l} \equiv \sqrt{\frac{2(l-2) !}{(l+2) !}}
$$

From Ref. [21], we can express the double derivatives in terms of spin-2 spherical harmonics ${ }_{ \pm 2} Y_{(l m)}(\hat{\mathbf{n}})$ as

$$
\begin{aligned}
Y_{(l m): a b}= & -\frac{l(l+1)}{2} Y_{(l m)} g_{a b} \\
& +\frac{1}{2} \sqrt{\frac{(l+2) !}{(l-2) !}}\left[Y_{(2 m)}\left(m_{+} \otimes m_{+}\right)\right. \\
& \left.+{ }_{-2} Y_{(l m)}\left(m_{-} \otimes m_{-}\right)\right]_{a b},
\end{aligned}
$$

where

$$
m_{ \pm}=\frac{1}{\sqrt{2}}\left(\hat{e}_{\theta} \mp i \hat{e}_{\phi}\right)
$$

Combining Eqs. (11) and (12) with Eqs. (8)-(10), we can express the tensor spherical harmonics $Y_{(l m)}^{E, B}$ in terms of spin-2 spherical harmonics as ${ }^{3}$

$$
Y_{(l m)}^{B}=\left(\begin{array}{cc}
\frac{i \sqrt{2}}{4}\left({ }_{+2} Y-{ }_{-2} Y\right) & \frac{\sqrt{2}}{4} \sin (\theta)\left({ }_{-2} Y+{ }_{+2} Y\right) \\
\frac{\sqrt{2}}{4} \sin (\theta)\left({ }_{-2} Y+{ }_{+2} Y\right) & \frac{i \sqrt{2}}{4} \sin ^{2}(\theta)\left({ }_{-2} Y-{ }_{+2} Y\right)
\end{array}\right),
$$

$Y_{(l m)}^{E}=\left(\begin{array}{cc}\frac{\sqrt{2}}{4}\left({ }_{+2} Y+{ }_{-2} Y\right) & \frac{i \sqrt{2}}{4} \sin (\theta)\left({ }_{-2} Y-{ }_{+2} Y\right) \\ \frac{i \sqrt{2}}{4} \sin (\theta)\left({ }_{-2} Y-{ }_{+2} Y\right) & -\frac{\sqrt{2}}{4} \sin ^{2}(\theta)\left({ }_{-2} Y+{ }_{+2} Y\right)\end{array}\right)$.

Using this result, we obtain

$$
\begin{aligned}
Y_{(l m)}^{B * a b} Y_{\left(l_{2} m_{2}\right) a b}^{B}= & \frac{1}{2}\left({ }_{-2} Y_{(l m)}^{*} \times{ }_{-2} Y_{\left(l_{2} m_{2}\right)}\right. \\
& \left.+{ }_{+2} Y_{(l m)}^{*} \times{ }_{+2} Y_{\left(l_{2} m_{2}\right)}\right),
\end{aligned}
$$

and

$$
\begin{aligned}
Y_{(l m)}^{E *, a b} Y_{\left(l_{2} m_{2}\right) a b}^{B}= & \frac{i}{2}\left({ }_{+2} Y_{(l m)}^{*} \times{ }_{+2} Y_{\left(l_{2} m_{2}\right)}\right. \\
& \left.-{ }_{-2} Y_{(l m)}^{*} \times{ }_{-2} Y_{\left(l_{2} m_{2}\right)}\right) .
\end{aligned}
$$

The next step is to use Eqs. (15) and (16) in order to rewrite the integrals in Eqs. (6) and (7) in terms of Wigner $3 \mathrm{j}$ symbols. We use Ref. [21], noting that the spin-spherical harmonics of zero spin are the regular spherical harmonics, ${ }_{0} Y_{(l m)} \equiv Y_{(l m)}$. Let us first look at the case of the induced $B$ mode, where we have

$$
\int d \hat{n} Y_{(l m)}^{B * a b} Y_{(L M)} Y_{\left(l_{2} m_{2}\right) a b}^{B}=\frac{1}{2}(-1)^{m} \sqrt{\frac{(2 l+1)(2 L+1)\left(2 l_{2}+1\right)}{4 \pi}}\left[\left(\begin{array}{ccc}
l & L & l_{2} \\
-2 & 0 & 2
\end{array}\right)+\left(\begin{array}{ccc}
l & L & l_{2} \\
2 & 0 & -2
\end{array}\right)\right]\left(\begin{array}{ccc}
l & L & l_{2} \\
-m & M & m_{2}
\end{array}\right) .
$$

We now define ${ }^{4}$

$$
\begin{aligned}
\xi_{l m l_{2} m_{2}}^{L M} \equiv & (-1)^{m} \\
& \times \sqrt{\frac{(2 l+1)(2 L+1)\left(2 l_{2}+1\right)}{4 \pi}}\left(\begin{array}{ccc}
l & L & l_{2} \\
-m & M & m_{2}
\end{array}\right)
\end{aligned}
$$

and

$$
H_{l l_{2}}^{L} \equiv\left(\begin{array}{ccc}
l & L & l_{2} \\
2 & 0 & -2
\end{array}\right)
$$

Also, note that, due to the properties of the Wigner $3 \mathrm{j}$

\footnotetext{
${ }^{3}$ We suppress the $(l m)$ indices for $Y$ in this formula.

${ }^{4}$ Note that the definitions of $\xi_{l m l^{\prime} m^{\prime}}^{L M}$ and $H_{l l^{\prime}}^{L}$ differ from those in Ref. [14]. We define these quantities in this way to avoid division by zero.
}

symbols, ${ }^{5}$ the sum in Eq. (17) vanishes, unless $l+l_{2}+$ $L=$ even. Replacing Eqs. (17)-(19), in Eq. (6), we come to a relatively simple expression for the rotation-induced $B$ mode,

$$
\delta B_{l m}=2 \sum_{L M} \sum_{l_{2} m_{2}} \alpha_{L M} E_{l_{2} m_{2}} \xi_{l m l_{2} m_{2}}^{L M} H_{l l_{2}}^{L}
$$

where the only nonzero terms in the sum are those that satisfy $l+l_{2}+L=$ even.

Similarly, for the case of the rotation-induced $E$ mode, again using the properties of the Wigner $3 \mathrm{j}$ symbols, ${ }^{6}$ we have

\footnotetext{
${ }^{5}$ See Appendix A: changing the sign on all three $m$ 's, brings up a factor of $(-1)^{l+l_{2}+L}$.

${ }^{6}$ See Appendix A: if the sum of $m$ 's does not vanish, the value of the symbol is zero.
} 


$$
\int d \hat{n} Y_{(l m)}^{E *, a b} Y_{(L M)} Y_{\left(l_{2} m_{2}\right) a b}^{B}=i \xi_{l m l_{2} m_{2}}^{L M} H_{l l_{2}}^{L},
$$

and then replacing this in Eq. (7), we get

$$
\delta E_{l m}=2 i \sum_{L M} \sum_{l_{2} m_{2}} \alpha_{L M} E_{l_{2} m_{2}} \xi_{l m l_{2} m_{2}}^{L M} H_{l l_{2}}^{L} .
$$

Note that in Eqs. (21) and (22) the only nonzero terms are those that satisfy $l+l_{2}+L=$ odd.

\section{B. Induced correlations}

Using the induced modes, derived in the previous subsection, we derive four correlations that are modified/induced by the rotation: $\mathrm{EB}, \mathrm{EE}, \mathrm{TB}$, and TE (there is also a BB correlation, but it is higher order in $\alpha$, and thus neglected). Every mode that we detect will contain the sum of the primordial (at the surface of last scatter) and the rotation-induced component,

$$
E_{l m}=E_{l m, 0}+\delta E_{l m}, \quad B_{l m}=\delta B_{l m} .
$$

We use the definitions of the EE and TE power spectra,

$$
\begin{aligned}
\left\langle E_{l m, 0} E_{l^{\prime} m^{\prime}, 0}^{*}\right\rangle & =C_{l}^{E E} \delta_{l l^{\prime}} \delta_{m m^{\prime}}, \\
\left\langle E_{l m, 0} T_{l^{\prime} m^{\prime}, 0}^{*}\right\rangle & =C_{l}^{T E} \delta_{l l^{\prime}} \delta_{m m^{\prime}} .
\end{aligned}
$$

Finally, we obtain the expressions for the four correlators, to first order in $\alpha$. Using Eqs. (20) and (22)-(24), we obtain

$$
\begin{aligned}
\left\langle B_{l m} E_{l^{\prime} m^{\prime}}^{*}\right\rangle= & 2 \sum_{L M} \alpha_{L M} C_{l^{\prime}}^{E E} \xi_{l m l^{\prime} m^{\prime}}^{L M} H_{l l^{\prime}}^{L} \\
= & \frac{1}{\sqrt{\pi}} \alpha_{00} C_{l^{\prime}}^{E E} \delta_{l l^{\prime}} \delta_{m m^{\prime}} \\
& +2 \sum_{L \geq 1} \sum_{M=-L}^{L} \alpha_{L M} C_{l^{\prime}}^{E E} \xi_{l m l^{\prime} m^{\prime}}^{L M} H_{l l^{\prime}}^{L},
\end{aligned}
$$

and the rest are

$$
\begin{aligned}
\left\langle E_{l m} E_{l^{\prime} m^{\prime}}^{*}\right\rangle= & C_{l}^{E E} \delta_{l l^{\prime}} \delta_{m m^{\prime}} \\
& +2 i \sum_{L M}\left(C_{l^{\prime}}^{E E}-C_{l}^{E E}\right) \alpha_{L M} \xi_{l m l^{\prime} m^{\prime}}^{L M} H_{l l^{\prime}}^{L}, \\
\left\langle B_{l m} T_{l^{\prime} m^{\prime}}^{*}\right\rangle=2 \sum_{L M} \alpha_{L M} C_{l^{\prime}}^{T E} \xi_{l m l^{\prime} m^{\prime}}^{L M} H_{l l^{\prime}}^{L}, & \\
\left\langle E_{l m} T_{l^{\prime} m^{\prime}}^{*}\right\rangle= & C_{l}^{T E} \delta_{l l^{\prime}} \delta_{m m^{\prime}}+2 i \sum_{L M} C_{l^{\prime}}^{T E} \alpha_{L M} \xi_{l m l^{\prime} m^{\prime}}^{L M} H_{l l^{\prime}}^{L}
\end{aligned}
$$

Note that for EB and TB, the sum is taken over the terms that satisfy $l+l^{\prime}+L=$ even and in EE and TE over $l+$ $l^{\prime}+L=$ odd.

\section{ESTIMATORS FOR THE ROTATION-ANGLE SPHERICAL-HARMONIC COEFFICIENTS}

If we assume that the primordial CMB temperature/ polarization pattern is a realization of a statistically isotropic Gaussian random field, then the spherical-harmonic coefficients $\left(T_{l m}, B_{l m}\right.$, or $\left.E_{l m}\right)$ for the primordial field are all uncorrelated. As Eqs. (25)-(28) show however, rotation induces off-diagonal correlations; i.e., correlations between spherical-harmonic coefficients of different $l m$ and $l^{\prime} m^{\prime}$.

While the correlations of specific $l m-l^{\prime} m^{\prime}$ pairs depend on the azimuthal quantum numbers $m$ and $m^{\prime}$, they can be parametrized in terms of the rotational invariants (i.e., independent of $m$ ),

$$
D_{l l^{\prime}}^{L M, A} \equiv 2 \alpha_{L M} Z_{l l^{\prime}}^{A} H_{l l^{\prime}}^{L},
$$

where the quantities $Z_{l l^{\prime}}^{A}$ are given in Table I; they are obtained from Eqs. (25)-(28). The EB correlator, for a given $l l^{\prime}$ pair with $l \neq l^{\prime}$, is different than the BE correlator. We thus consider both $\mathrm{BE}$ and $\mathrm{EB}$ (and similarly for TE/ET and TB/BT) and then restrict our sums to $l^{\prime} \geq l$ to avoid double-counting. We thus have for $l>l^{\prime}, A=$ $\{B E, E B, T B, B T, T E, E T, E E\}$, while for $l=l^{\prime}$, we have $A=\{B E, T E, T B, E E\}$. With these shorthands, the part of the $X X^{\prime}$ correlators (for $\left\{X, X^{\prime}\right\}=\{T, B, E\}$ ) can be written as

$$
\left\langle X_{l m}\left(X_{l^{\prime} m^{\prime}}^{\prime}\right)^{*}\right\rangle=\sum_{L M} D_{l l^{\prime}}^{L M, X X^{\prime}} \xi_{l m l^{\prime} m^{\prime}}^{L M}
$$

We now suppose that we have spherical-harmonic coefficients $T_{l m}^{\mathrm{map}}, E_{l m}^{\mathrm{map}}, B_{l m}^{\mathrm{map}}$, obtained from a CMB temperature/polarization map. These receive contributions from the true signal on the sky, reduced by the $l$-space window function $W_{l}=\exp \left(-l^{2} \sigma_{b}^{2} / 2\right)$, where $\sigma_{b}=$ $\theta_{\text {fwhm }} / \sqrt{8 \ln 2}=0.00741\left(\theta_{\text {fwhm }} / 1^{\circ}\right)$ for a Gaussian beam of width $\theta_{\text {fwhm }}$, and a contribution from detector noise. The predictions for the rotational invariants for the map are $D_{l l^{\prime}}^{L M, X X^{\prime}, \text { map }}=D_{l l^{\prime}}^{L M, X X^{\prime}} W_{l} W_{l^{\prime}}$. Following Refs. [14,19,22],

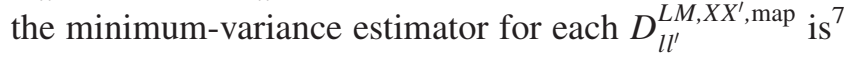

$$
\hat{D}_{l l^{\prime}}^{L M, X X^{\prime}, \mathrm{map}}=\left(G_{l l^{\prime}}^{L}\right)^{-1} \sum_{m m^{\prime}} X_{l m}^{\mathrm{map}} X_{l^{\prime} m^{\prime}}^{\prime, \mathrm{map}, *} \xi_{l m l^{\prime} m^{\prime}}^{L M},
$$

where we have used (see Appendix A),

$$
\sum_{m m^{\prime}}\left(\xi_{l m l^{\prime} m^{\prime}}^{L M}\right)^{2}=G_{l l^{\prime}}^{L} \equiv \frac{(2 l+1)\left(2 l^{\prime}+1\right)}{4 \pi} .
$$

Recall also that for EB and TB, only the terms in Eq. (31) that satisfy $l+l^{\prime}+L=$ even are nonvanishing, while for EE and TE only $l+l^{\prime}+L=$ odd terms are nonvanishing.

\footnotetext{
${ }^{7}$ Note that the definition of $G_{l l^{\prime}}^{L}$ differs from that in Refs. [14,19].
} 
TABLE I. The quantities $Z_{l l^{\prime}}^{A}$, defined in Eq. (29), for the various modes $\mathrm{A}$.

\begin{tabular}{lc}
\hline \hline $\mathrm{A}$ & $Z_{l l^{\prime}}^{A}$ \\
\hline $\mathrm{BE}$ & $C_{l^{\prime}}^{E E}$ \\
$\mathrm{~EB}$ & $C_{l}^{E E}$ \\
$\mathrm{EE}$ & $-i\left(C_{l^{\prime}}^{E E}-C_{l}^{E E}\right)$ \\
$\mathrm{BT}$ & $C_{l^{\prime}}^{T E}$ \\
$\mathrm{~TB}$ & $C_{l}^{T E}$ \\
$\mathrm{ET}$ & $-i C_{l^{\prime}}^{T E}$ \\
$\mathrm{TE}$ & $-i C_{l}^{T E}$ \\
\hline \hline
\end{tabular}

The variances with which each $\hat{D}_{l l^{\prime}}^{L M, X X^{\prime} \text {, map }}$ can be measured can also be calculated. Moreover, measurements of

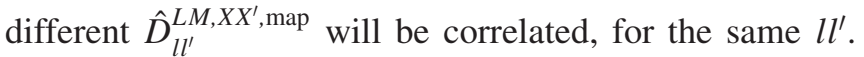

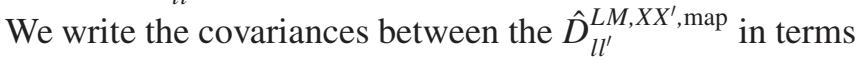
of the quantities,

$$
\begin{aligned}
\mathcal{C}_{A A^{\prime}}^{l l^{\prime}} \equiv & G_{l l^{\prime}}^{L}\left(\left\langle\hat{D}_{l l^{\prime}}^{L M, A, \text { map }} \hat{D}_{l l^{\prime}}^{L M, A^{\prime}, \text { map }}\right\rangle\right. \\
& \left.-\left\langle\hat{D}_{l l^{\prime}}^{L M, A, \text { map }}\right\rangle\left\langle\hat{D}_{l l^{\prime}}^{L M, A^{\prime} \text { map }}\right\rangle\right) .
\end{aligned}
$$

In principle, $\mathcal{C}_{A A^{\prime}}^{l l^{\prime}}$ is a $7 \times 7$ matrix (in the $A A^{\prime}$ space) for $l \neq l^{\prime}$ and $4 \times 4$ for $l=l^{\prime}$. However, the matrix is sparsely populated in the $A A^{\prime}$ space; it can be written in blockdiagonal form, since the EB and TB correlators are nonvanishing only for $l+l^{\prime}+L=$ even while those for TE and EE are nonvanishing only for $l+l^{\prime}+L=$ odd. We present explicit expressions for the relevant entries of $\mathcal{C}_{A A^{\prime}} l^{\prime}$ below.

\section{A. Minimum-variance estimators for the rotation-angle coefficients}

We now write down the minimum-variance quadratic estimator that can be constructed for the rotation-angle coefficients $\alpha_{L M}$ from the measured temperature/polarization map.

To begin, we recall that each $\hat{D}_{l l^{\prime}}^{L M, A \text {, map }}$ (i.e., each $l l^{\prime}$ and $A A^{\prime}$, for a given $L M$ ) provides a measurement of $\alpha_{L M}$, through $\left(\hat{\alpha}_{L M}\right)_{l l^{\prime}}^{A}=\hat{D}_{l l^{\prime}}^{L M, A, \text { map }} / F_{l l^{\prime}}^{L, A}$, in terms of the quantities

$$
F_{l l^{\prime}}^{L, A} \equiv 2 Z_{l l^{\prime}}^{A} H_{l l^{\prime}}^{L} W_{l} W_{l^{\prime}}
$$

The variances and covariances of the $\left(\hat{\alpha}_{L M}\right)_{l l^{\prime}}^{A}$ are given simply in terms of those for $D_{l l}^{L M, A \text {,map }}$ scaled by the appropriate factors of $F^{L, A} l^{\prime}$.

The minimum-variance estimator $\hat{\alpha}_{L M}$ is then obtained by summing all of the individual estimators, for a given $L M$, with inverse-variance weighting (and taking into account also the covariances). The minimum-variance estimator is thus

$$
\hat{\alpha}_{L M}=\sigma_{\alpha_{L M}}^{2} \sum_{l^{\prime} \geq l} G_{l l^{\prime}}^{L} \sum_{A A^{\prime}} F_{l l^{\prime}}^{L, A^{\prime}} \hat{D}_{l l^{\prime}}^{L M, A, \operatorname{map}}\left[\left(\mathcal{C}^{l l^{\prime}}\right)^{-1}\right]_{A A^{\prime}}
$$

and it has variance $\sigma_{\alpha_{L M}}^{2}$ given by

$$
\sigma_{\alpha_{L M}}^{-2}=\sum_{l^{\prime} \geq l} G_{l l^{\prime}}^{L} \sum_{A A^{\prime}} F_{l l^{\prime}}^{L, A} F_{l l^{\prime}}^{L, A^{\prime}}\left[\left(\mathcal{C}^{l l^{\prime}}\right)^{-1}\right]_{A A^{\prime}}
$$

In these expressions, the $A A^{\prime}$ sums are over $\{E B, B E, T B, B T, E E, T E, E T\} \quad$ for $\quad l \neq l^{\prime} \quad$ and $\{E B, T B, E E, T E\}$ for $l=l^{\prime}$, and the matrix inversion is in the $A A^{\prime}$ space.

\section{B. Variance for $D$}

We assume that the noise is isotropic and that it is not correlated with the data; that the temperature and polarization noises are not correlated; and that the temperature/ polarization noises between different pixels are not correlated. If so, then the power spectra for the map are $C_{l}^{A \text {,map }}=C_{l}^{A} W_{l}^{2}+C_{l}^{A \text {,noise }}$, where here $\mathrm{A}=\{\mathrm{TT}, \mathrm{EE}, \mathrm{BB}\}$, as described in Ref. [14]. The TT, EE, and BB noise power spectra are

$$
\begin{aligned}
& C_{l}^{T T, \text { noise }}=\left(4 \pi / N_{\mathrm{pix}}\right) \sigma_{T}^{2}, \\
& C_{l}^{B B, \text { noise }}=C_{l}^{E E, \text { noise }}=\left(4 \pi / N_{\mathrm{pix}}\right) \sigma_{P}^{2},
\end{aligned}
$$

where $\sigma_{T}^{2}$ and $\sigma_{P}^{2}$ are the temperature and noise variances in each pixel, and $N_{\text {pix }}$ is the number of pixels in the map. The instrumental-noise contributions to the cross-power spectra are zero:

$$
C_{l}^{E B, \text { noise }}=C_{l}^{T B \text {,noise }}=C_{l}^{T E \text {,noise }}=0 .
$$

Now we can calculate the desired variances for $D$, using Eqs. (31) and the power spectra $C_{l}^{A}$. For EB, for $l \neq l^{\prime}$, we have

$$
\begin{gathered}
\mathcal{C}_{B E, B E}^{l l^{\prime}}=C_{l}^{B B, \text { map }} C_{l^{\prime}}^{E E \text { map }}, \quad \mathcal{C}_{E B, E B}^{l l^{\prime}}=C_{l^{\prime}}^{B B, \text { map }} C_{l}^{E E, \text { map }}, \\
\mathcal{C}_{E B, B E}^{l l^{l^{\prime}}}=\mathcal{C}_{B E, E B}^{l l^{\prime}}=0
\end{gathered}
$$

and for $l=l^{\prime}$,

$$
\mathcal{C}_{B E, B E}^{l l}=C_{l}^{B B \text {,map }} C_{l}^{E E \text {,map }} \text {. }
$$

The covariances for BT and TB are the same, with the replacements $\mathrm{E} \rightarrow \mathrm{T}$. There are also covariances between the TB and EB estimators. For $l=l^{\prime}$, they are

$$
\mathcal{C}_{B E, B T}^{l l}=C_{l}^{B B \text {,map }} C_{l}^{T E \text { map }} .
$$

For $l \neq l^{\prime}$,

$$
\begin{gathered}
\mathcal{C}_{B E, B T}^{l l^{\prime}}=C_{l}^{B B, \text { map }} C_{l^{\prime}}^{T E \text {,map }}, \quad \mathcal{C}_{E B, T B}^{l l^{\prime}}=C_{l^{\prime}}^{B B, \text { map }} C_{l}^{T E, \text { map }}, \\
\mathcal{C}_{B E, T B}^{l l^{\prime}}=\mathcal{C}_{E B, B T}^{l l^{\prime}}=0 .
\end{gathered}
$$

For EE,

$$
\mathcal{C}_{E E, E E}^{l l^{\prime}}=\left(1+\delta_{l l^{\prime}}\right) C_{l}^{E E, \text { map }} C_{l^{\prime}}^{E E \text { map }}
$$


For the TE case, for $l \neq l^{\prime}$, we have

$$
\begin{gathered}
\mathcal{C}_{T E, T E}^{l l^{\prime}}=C_{l}^{T T, \text { map }} C_{l^{\prime}}^{E E \text { map }}, \quad \mathcal{C}_{E T, E T}^{l l^{\prime}}=C_{l^{\prime}}^{T T \text {,map }} C_{l}^{E E \text {,map },} \\
\mathcal{C}_{T E, E T}^{l l^{\prime}}=\mathcal{C}_{E T, T E}^{l l^{\prime}}=C_{l}^{T E \text {,map }} C_{l^{\prime}}^{T E \text { map }},
\end{gathered}
$$

and for $l=l^{\prime}$,

$$
\mathcal{C}_{E T, E T}^{l l}=C_{l}^{T T, \text { map }} C_{l}^{E E \text {,map }}\left(C_{l}^{T E \text {,map }}\right)^{2} .
$$

There are also TE-EE covariances. However, since TE and $\mathrm{EE}$ are almost always weaker probes of the rotation, we do not include these additional expressions here.

\section{Quadratic estimators for BE (or TE) only}

As an example (and for clarity), we can write down the expressions for the estimator and noise in the case where we use only information from the BE correlator to determine $\alpha_{L M}$. In this case, the estimator is

$$
\begin{aligned}
\hat{\alpha}_{L M}= & \sigma_{\alpha_{L M}}^{2} \sum_{l^{\prime} \geq l}\left(1+\delta_{l l^{\prime}}\right)^{-1} G_{l l^{\prime}}^{L}\left[\frac{F_{l l^{\prime}}^{L, B E} D_{l l^{\prime}}^{L M, B E, \text { map }}}{C_{l}^{B B, \text { map }} C_{l^{\prime}}^{E E \text { map }}}\right. \\
& +(\mathrm{B} \leftrightarrow \mathrm{E})],
\end{aligned}
$$

and the noise is given by

$$
\sigma_{\alpha_{L M}}^{-2}=\sum_{l^{\prime} \geq l}\left(1+\delta_{l l^{\prime}}\right)^{-1} G_{l l^{\prime}}^{L}\left[\frac{\left(F_{l l^{\prime}}^{L, B E}\right)^{2}}{C_{l}^{B B, \text { map }} C_{l^{\prime}}^{E E \text { map }}}+(\mathrm{B} \leftrightarrow \mathrm{E})\right] .
$$

The $l l^{\prime}$ sums here are over values that satisfy $l+l^{\prime}+L=$ even. The estimator and variance for TB are the same after the replacement $\mathrm{E} \rightarrow \mathrm{T}$.

\section{FLAT-SKY LIMIT AND COMPARISON WITH PREVIOUS WORK}

In this section, we derive the flat-sky limit of the variances for the rotation on the full sky and compare our results to those of Ref. [15]. We work out the EB case, where $l+l^{\prime}+L=$ even. The other three cases follow analogously.

From Eqs. (47) and (34), the variance is

$$
\begin{aligned}
\sigma_{L}^{-2}= & 4 \sum_{l^{\prime}>l}\left(H_{l l^{\prime}}^{L}\right)^{2} G_{l l^{\prime}}^{L}\left(W_{l} W_{l^{\prime}}\right)^{2}\left[\frac{\left(C_{l^{\prime}}^{E E}\right)^{2}}{C_{l}^{B B, \text { map }} C_{l^{\prime}}^{E E \text { map }}}\right. \\
& +\frac{\left(C_{l}^{E E}\right)^{2}}{\left.C_{l^{\prime}}^{B B \text { map }} C_{l}^{E E \text { map }}\right] .}
\end{aligned}
$$

The two terms in Eq. (48) are the same under the exchange of $l$ and $l^{\prime}$. Thus, after renaming the indices on one of the two terms, we get the sums over $l<l^{\prime}$ and $l>l^{\prime}$, which covers the whole range of $l$ 's. ${ }^{8}$ We are left with

\footnotetext{
${ }^{8}$ Note that, when we switch to integration, as shown further on in the text, the $l=l^{\prime}$ term is of measure zero, and can be ignored.
}

$$
\left(\sigma_{L}\right)^{-2}=4 \sum_{l l^{\prime}} X_{l l^{\prime}}^{L} G_{l l^{\prime}}^{L}\left(H_{l l^{\prime}}^{L}\right)^{2}
$$

where we have defined

$$
X_{l l^{\prime}}^{L} \equiv\left(W_{l} W_{l^{\prime}}\right)^{2} \frac{\left(C_{l^{\prime}}^{E E}\right)^{2}}{C_{l^{\prime}}^{E E, \text { map }} C_{l}^{B B, \text { map }}} .
$$

We now derive the limit of high multipoles. ${ }^{9}$ We start by using the approximation [21],

$$
H_{l l^{\prime}}^{L} \approx \cos 2 \varphi_{l l^{\prime}}\left(\begin{array}{ccc}
l & l^{\prime} & L \\
0 & 0 & 0
\end{array}\right)
$$

for the $L+l+l^{\prime}=$ even case. From Eqs. (32) and (51), we have

$$
G_{l l^{\prime}}^{L}\left(H_{l l^{\prime}}^{L}\right)^{2} \stackrel{L, l, l^{\prime} \rightarrow \infty}{\rightarrow} \frac{l l^{\prime}}{\pi}\left(\begin{array}{ccc}
l & l^{\prime} & L \\
0 & 0 & 0
\end{array}\right)^{2} \cos ^{2} 2 \varphi_{l l^{\prime}}
$$

From the relation of the spherical harmonics and Wigner $3 \mathrm{j}$ symbols (see Appendix A), this gives, for large $L$,

$$
\left(\sigma_{L}\right)^{-2} \underset{L, l, l^{\prime} \rightarrow \infty}{\longrightarrow} 4 \sum_{l l^{\prime}} X_{l l^{\prime}}^{L} \sqrt{\frac{l l^{\prime}}{2 \pi L}} \int d \hat{n} Y_{l 0} Y_{l^{\prime} 0} Y_{L 0} \cos ^{2} 2 \varphi_{l l^{\prime}}
$$

Given that

$$
\int_{0}^{2 \pi} \frac{d \varphi_{l}}{2 \pi} e^{i m \varphi_{l}}=\delta_{m, 0}
$$

Equation (53) can be rewritten using

$$
\begin{aligned}
& \sum_{m m^{\prime} M} \sum_{l l^{\prime}} \sqrt{\frac{l l^{\prime}}{2 \pi L} \int d \hat{n} Y_{l m}^{*} Y_{l^{\prime} m^{\prime}} Y_{L M} \delta_{M, 0} \delta_{m, 0} \delta_{m^{\prime}, 0}} \\
& =\sum_{m m^{\prime} M} \sum_{l l^{\prime}} \sqrt{\frac{l l^{\prime}}{2 \pi L}} \int d \hat{n} Y_{l m}^{*} Y_{l^{\prime} m^{\prime}} Y_{L M} \\
& \quad \times \int \frac{d \varphi_{l} d \varphi_{l^{\prime}} d \varphi_{L}}{(2 \pi)^{3}} e^{i\left(M \varphi_{L}+m^{\prime} \varphi_{l^{\prime}}-m \varphi_{l}\right)} .
\end{aligned}
$$

We use relations from Ref. [21]

$$
\begin{aligned}
e^{i \cdot \vec{l} \cdot \vec{n}} \approx & \sqrt{\frac{2 \pi}{l} \sum_{m} i^{m} Y_{l m} e^{i m \varphi_{l}},} \\
\delta\left(\vec{L}-\left(\vec{l}-\vec{l}^{\prime}\right)\right)= & \int \frac{d \hat{n}}{(2 \pi)^{2}} e^{i\left(\vec{L}-\vec{l}+\vec{l}^{\prime}\right) \cdot \hat{n}} \\
\approx & \int \frac{d \hat{n}}{(2 \pi)^{2}} \sum_{m m^{\prime} M} Y_{l m}^{*} Y_{l^{\prime} m^{\prime}} Y_{L M} \sqrt{\frac{(2 \pi)^{3}}{l l^{\prime} L}} \\
& \times e^{i\left(M \varphi_{L}+m^{\prime} \varphi_{l^{\prime}}-m \varphi_{l}\right)},
\end{aligned}
$$

and replace the sum with the integral,

\footnotetext{
${ }^{9}$ If $L$ is large, then the triangle inequalities and the requirement for nonflat triangles ensures that $l$ and $l^{\prime}$ are also large.
} 


$$
\sum_{l l^{\prime}} \int d \varphi_{l} d \varphi_{l^{\prime}} l l^{\prime} \leftrightarrow \iint d^{2} \vec{l} d^{2} \vec{l}^{\prime} .
$$

From Eqs. (55)-(58) and (53), and after integrating over $d \varphi_{L}$, we obtain the flat-sky limit for variance in the EB case,

$\left(\sigma_{L}\right)^{-2} \underset{L, l, l^{\prime} \rightarrow \infty}{\rightarrow} 4 \int \frac{d^{2} l^{\prime}}{(2 \pi)^{2}} \cos ^{2} 2 \varphi_{l^{\prime} l}\left(W_{l} W_{l^{\prime}}\right)^{2} \frac{\left(C_{l^{\prime}}^{E E}\right)^{2}}{C_{l}^{B B, \text { map }} C_{l^{\prime}}^{E E, \text { map }} . ~}$

This can be shown to agree with the results of Ref. [15] after combining their Eqs. (7) and (8) and results from their Table I.

\section{NUMERICAL RESULTS}

We now present numerical results for the variances of the estimators for a position-dependent rotation, for different instruments. The primordial power spectra, at the surface of last scatter, are obtained using WMAP-5 cosmological parameters: $\Omega_{b} h^{2}=0.02267, \Omega_{c} h^{2}=0.1131$, $\Lambda=0.726, n_{s}=0.960, \tau=0.084$, and a power spectrum normalized to WMAP5 [11].

We analyze three different experiments: (i) CMBPol's (EPIC-2m) $150 \mathrm{GHz}$ channel with resolution $\theta_{\mathrm{fwhm}}=5^{\prime}$, taking the relevant parameters as given in Ref. [23], a noise-equivalent temperature $\mathrm{NET}=2.8 \mu \mathrm{K} \sqrt{\mathrm{sec}}$ and the observation time $t_{\mathrm{obs}}=4 \mathrm{yr}$; (ii) Planck $143 \mathrm{GHz}$ channel, with $\theta_{\text {fwhm }}=7.1^{\prime}, \quad \mathrm{NET}=31 \mu \mathrm{K} \sqrt{\mathrm{sec}}$ and $t_{\mathrm{obs}}=1.2 \mathrm{yr}$; (iii) WMAP, with $\theta_{\text {fwhm }}=21^{\prime}, \quad \sigma_{T}=$ $30 \mu \mathrm{K}$ and $\sigma_{P}=42.6 \mu \mathrm{K}$ [19]. The NET parameters specified for Planck and CMBPol are related to the tem-
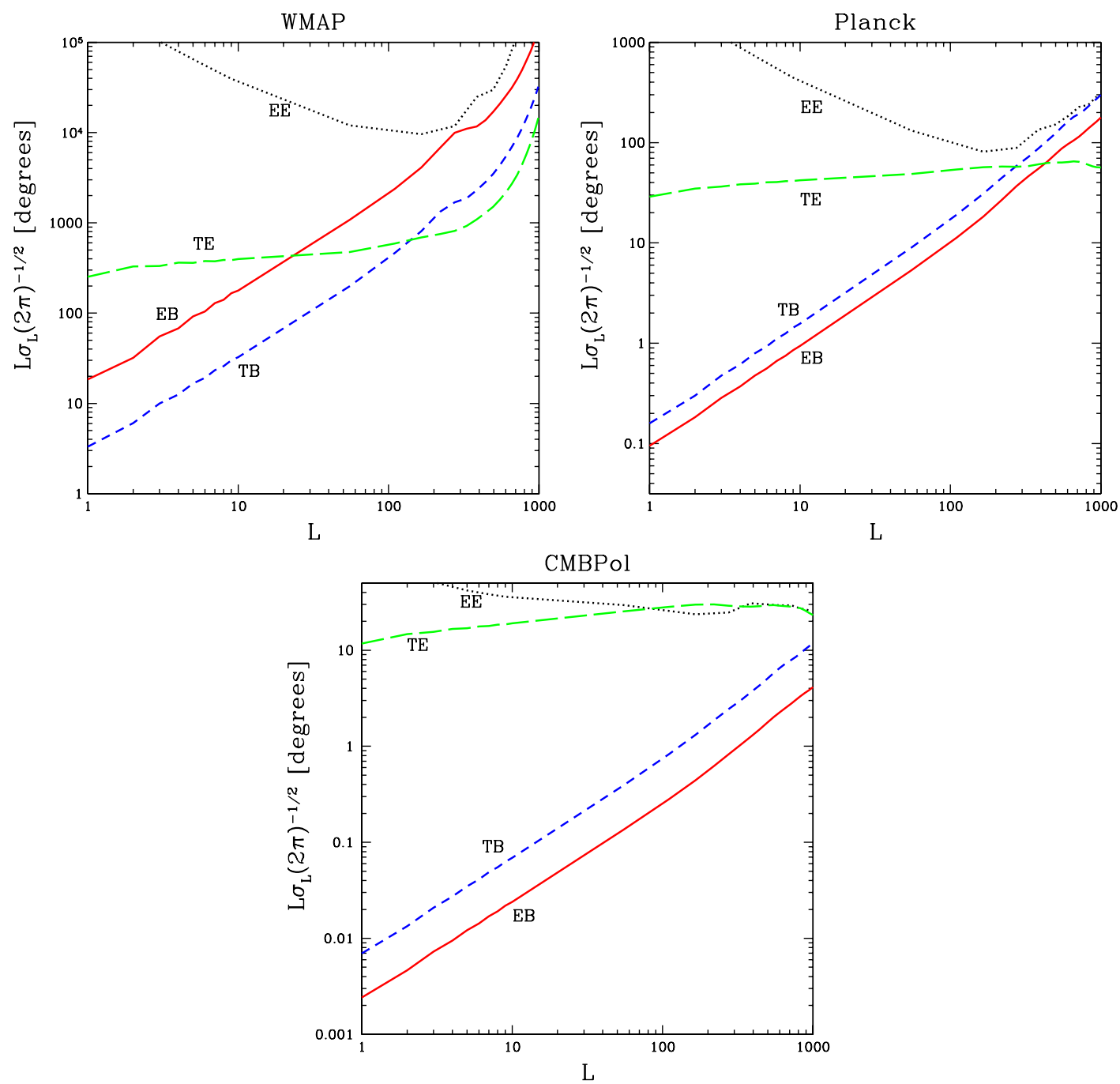

FIG. 1 (color online). Variances for the four rotation estimators for WMAP, Planck, and CMBPol are shown. We see that, at low multipoles, the lowest-error estimator for Planck and CMBPol comes from the EB correlation, and for WMAP from the TB correlation. 

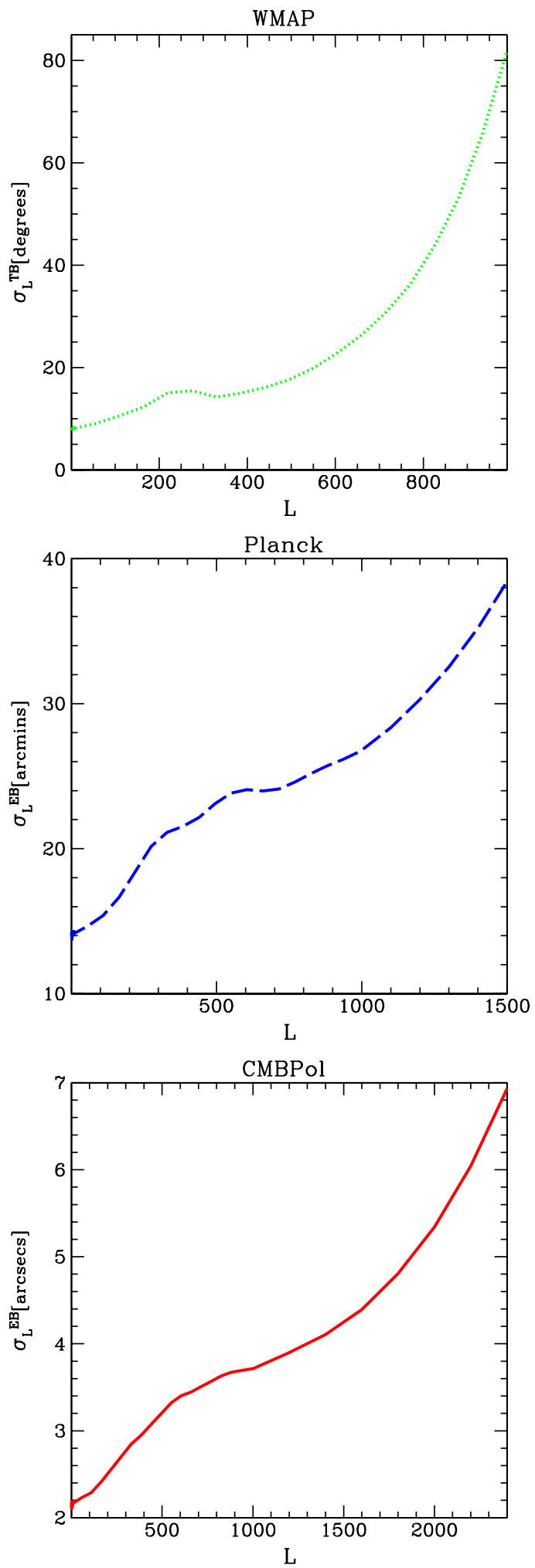

FIG. 2 (color online). The variances of the estimators for rotation are shown in linear scale, for WMAP (variance from the TB correlator), Planck (from EB) and CMBPol (from EB). The range of the plots is chosen so that it covers the resolution domain of the instrument. The exponential rise at high $L$, due to the window function, is visible. Note the difference in scale on the $y$-axis in all three panels.

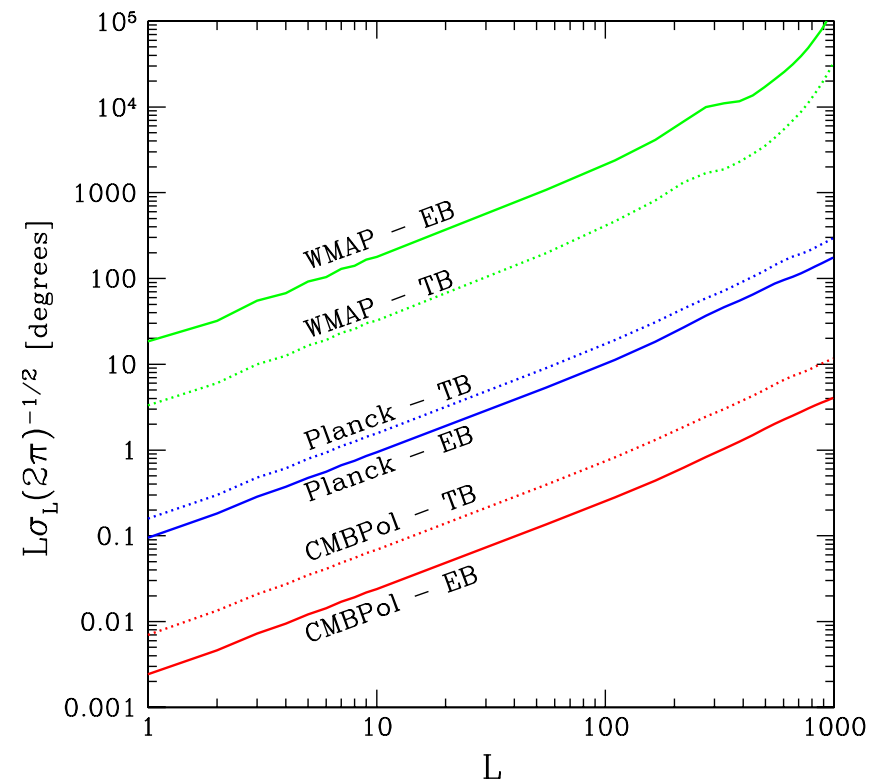

FIG. 3 (color online). The best constraints to rotation, i.e. the variances for estimators from the EB and TB correlations, are compared for three instruments. Planck gives about 2 orders of magnitude improvement in comparison to WMAP, and CMBPol is one order-of-magnitude better than Planck.

perature/polarization pixel-noise variances through $\sigma_{T}^{2} / N_{\text {pix }}=f_{\text {sky }}(\mathrm{NET})^{2} / t_{\text {obs }}$, where $f_{\text {sky }}$ is the fractional sky coverage (here assumed to be unity).

We evaluate the expressions for variances derived in the previous section, for each of the three experiments, and show in Fig. 1 the numerical results for EB, EE, TB, and TE correlations.

The first thing to notice about the variance levels in these three experiments is that both the lower noise and the higher resolution contribute to about 2 orders of magnitude improvement in the sensitivity to a rotation from WMAP to Planck and will lead to yet more than another order-ofmagnitude improvement in CMBPol. This is illustrated in more detail in Fig. 3, where the variances in the EB and TB estimators are compared for all three experiments.

From Fig. 1, we see that, at low multipoles (below $L$ of about 200), the most sensitive estimators for all three experiments will be those derived from the EB and TB correlations. This comes about because of the absence of any TB or EB correlation under the assumption (justified largely by upper limits to the $B$ mode amplitude) of no $B$ modes at the surface of last scatter. We separately look at the TB-estimator variance for WMAP and the EBestimator variances for Planck and CMBPol, in Fig. 2.

For WMAP, we find a TB-estimator variance of $8.3^{\circ}$ at $L=0$, which is consistent with the current constraints on a uniform rotation ${ }^{10}[10,11]$ (see Appendix C). The dipole

\footnotetext{
${ }^{10}$ Note that we calculate the multipole moments of rotation, so a uniform rotation of angle $\alpha$ has $\alpha_{00}=\sqrt{4 \pi} \alpha$. Thus, $\alpha_{00}=$ $8.3^{\circ}$ is equivalent to $\alpha=2.3^{\circ}$.
} 


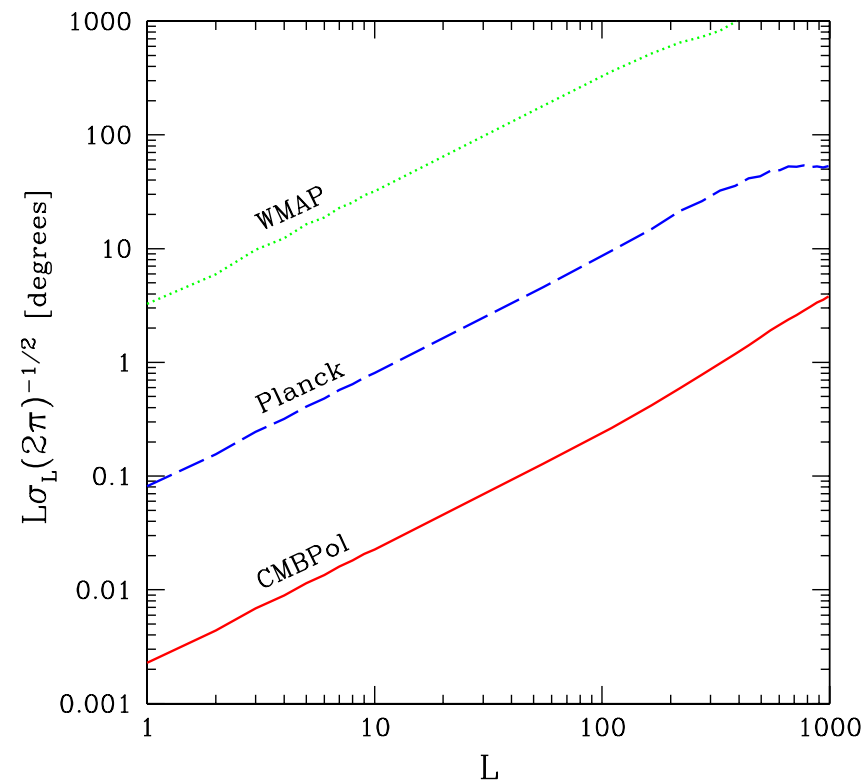

FIG. 4 (color online). The combined variance, for all four estimators for rotation, is shown for WMAP, Planck and CMBPol. Note that, due to the inverse-weight summing, the smallest of the four variances dominates the shape of the curves.

and quadrupole components of the rotation have the same variances as the monopole $(L=0)$, since the variance remains fairly flat out to $L$ of about 100 , in all three instruments. Above $L \simeq 400$, the variance increases rapidly. This happens when the exponential part of the window functions dominates (due to the finite angular resolution of the instrument). Also, since the correlation angle for polarization is about 10 times smaller than that for temperature, the exponential tail in the EE-estimator case becomes prominent at higher multipoles than in the TE case. The variance from the EB-estimator at $L=0$ is $46.2^{\circ}$ for WMAP, and thus not constraining. The TE-estimator variance quickly drops below the EB-estimator variance (at $L \simeq 20$ ) and below the TB-estimator variance (at $L \simeq 150$ ). However, the variance $\sigma_{L}$ at these $L$ is so large ( $\gtrsim 100$ ) that the measurements are not at all constraining. Similar features are apparent in plots for the other two experiments.

For Planck, the variances of the EB and TB estimators are more comparable, and the constraints to all rotation multipoles in the range from $L$ of 0 to about 300 come from the EB variance. At $L=0$ the variance is $14^{\prime}$ and $24^{\prime}$, for the EB- and TB-estimator variances, respectively. Planck can thus provide an order-of-magnitude better sensitivity to the uniform rotation than the current WMAP sensitivity. For high multipoles, above $L \simeq 400$ or so, the TE-estimator variance becomes the smallest one. At $L \simeq 800$, a rapid rise in all four variances is visible, due to the limitations in angular resolution and the correlation angle of the polarization.

For CMBPol, the EB-estimator variance is the smallest in the whole range of multipoles from 0 to 1000 . At $L=0$,

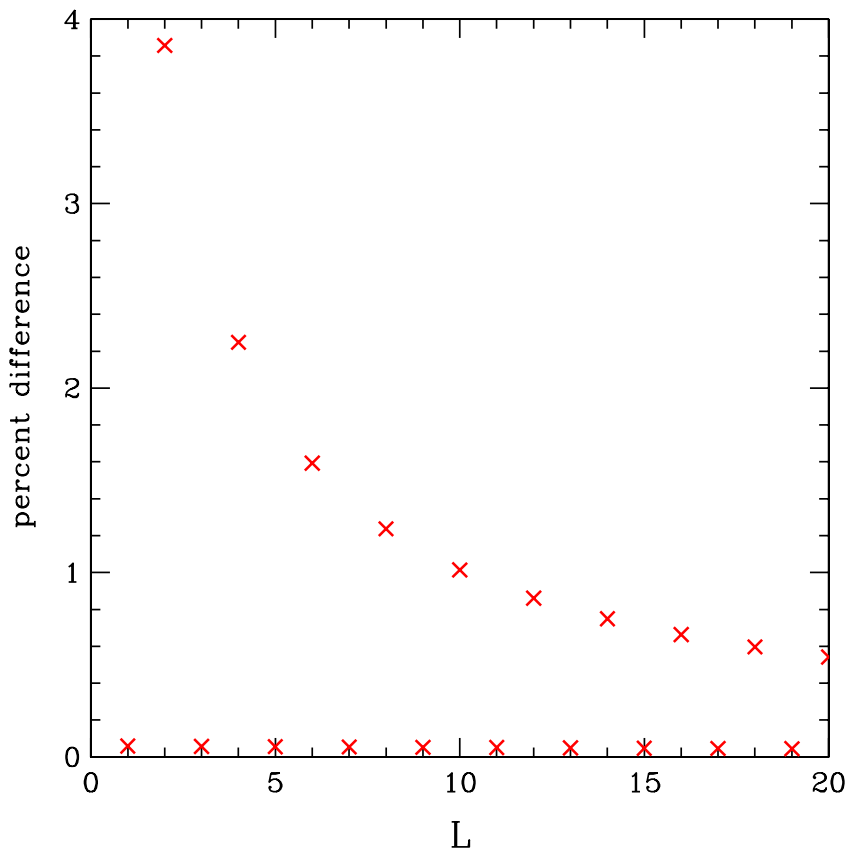

FIG. 5 (color online). Comparison between the full- and flatsky expressions for the variance in the low- $L$ regime. The discrepancy between the two is larger for even $L$ because of the additional contributions to the estimators from $l=l^{\prime}$.

the EB and TB values are, respectively, $2.2^{\prime \prime}$ and $6.3^{\prime \prime}$, which is better than Planck by more than an order-ofmagnitude. Similarly to WMAP and Planck variances, we observe a rapid rise in the variance above $L \simeq 1000$, corresponding to the resolution limitations and/or the polarization correlation length.

In Fig. 4 we show the variance for the combined minimum-variance estimator, obtained from all four estimators, with inverse-variance weighting. We have not included the covariance between the four estimators in this numerical calculation. However, this omission should make negligible difference for the WMAP and CMBPol curves, where the variance is determined primarily by $\mathrm{TB}$ and EB, respectively; the Planck curve may be increased, but only slightly. The run of this combined variance with $L$ differs very little from the smallest/constraining variance (that is TB in the case of WMAP and EB in the case of Planck and CMBPol), because that term dominates the sum.

As a check, we repeated our calculations for the EBcorrelator using the flat-sky formulas from Sec. IV, and compared those results to the full-sky. The two sets of variances are in good agreement at high multipoles, where the flat sky makes a valid approximation (better than a fraction of a percent for $L \gtrsim 50$ ). The discrepancy increases up to $\sim 4 \%$ at lower $L$. Figure 5 compares the variances obtained from the full- and flat-sky treatments at low $L$. Finally, we redid the full-sky calculations using the same instrumental parameters as in Ref. [15]; these are 
also in good agreement with our results at high L's (better than $7 \%$ for $L \gtrsim 50$, and about $11 \%$ at $L=0$ ).

\section{CONCLUSIONS AND SUMMARY}

Exotic mechanisms, such as quintessence fields that couple to the pseudoscalar of electromagnetism, could rotate the linear polarization of the CMB. In this paper, we derive the complete set of minimum-variance estimators for a position-dependent rotation of the CMB polarization, and thus provide a recipe, given a full-sky map, for measuring the rotation angle as a function of position on the sky. We also evaluate the variances with which the rotation-angle spherical-harmonic coefficients can be measured for WMAP, Planck, and CMBPol. Our results indicate that $\mathrm{EB}$ and $\mathrm{TB}$ correlations will provide more sensitive probes of the rotation angle than TE and EE correlations, and that EB becomes better, relative to TB, as the instrumental noise is reduced. We have checked that our results for the variances recover prior results, both analytically and numerically, in the flat-sky limit. As an additional check, Appendix $\mathrm{C}$ shows that our expressions for the rotation-angle variance recovers that expected for a uniform rotation. Appendix B shows that parity considerations can be used to distinguish the effects of rotation and of weak lensing on the $\mathrm{CMB}$ temperature/polarization map.

Now that we have elucidated the all-sky formalism, the next step will be to work out algorithms for recovery of the rotation angle for a map with partial sky coverage. We anticipate that analogous techniques for determining the cosmic-shear deflection angle may be adapted for this purpose.

\section{ACKNOWLEDGMENTS}

V. G. thanks Itamar Yaakov and Michael Kesden for useful comments. This work was supported by DoE DEFG03-92-ER40701 and the Gordon and Betty Moore Foundation.

\section{APPENDIX A: SOME USEFUL FORMULAS}

Some formulas used in Secs. II and III are included here. The metric tensor and its inverse on a unit 2-sphere (i.e. on the $s k y$ ) is

$$
\begin{gathered}
g=\left(\begin{array}{cc}
1 & 0 \\
0 & \sin ^{2} \theta
\end{array}\right), \\
g^{-1}=\left(\begin{array}{cc}
1 & 0 \\
0 & \frac{1}{\sin ^{2} \theta}
\end{array}\right) .
\end{gathered}
$$

The Levi-Civita tensor on a unit 2-sphere is

$$
\varepsilon=\left(\begin{array}{cc}
0 & \sin \theta \\
-\sin \theta & 0
\end{array}\right) .
$$

An orthonormal basis on a unit 2-sphere is

$$
\hat{e}_{\theta}=\left(\begin{array}{l}
1 \\
0
\end{array}\right), \quad \hat{e}_{\phi}=\left(\begin{array}{c}
1 \\
\sin \theta
\end{array}\right) .
$$

Some useful properties of the Wigner $3 \mathrm{j}$ symbols and related quantities include

$$
\begin{aligned}
& \left(\begin{array}{ccc}
l_{1} & l_{2} & l_{3} \\
m_{1} & m_{2} & m_{3}
\end{array}\right)=(-1)^{l_{1}+l_{2}+l_{3}}\left(\begin{array}{ccc}
l_{1} & l_{2} & l_{3} \\
-m_{1} & -m_{2} & -m_{3}
\end{array}\right), \\
& \left(\begin{array}{ccc}
l_{1} & l_{2} & l_{3} \\
m_{1} & m_{2} & m_{3}
\end{array}\right)=\left(\begin{array}{ccc}
l_{2} & l_{3} & l_{1} \\
m_{2} & m_{3} & m_{1}
\end{array}\right), \\
& \left(\begin{array}{ccc}
l_{1} & l_{2} & l_{3} \\
m_{1} & m_{2} & m_{3}
\end{array}\right)=(-1)^{l_{1}+l_{2}+l_{3}}\left(\begin{array}{ccc}
l_{2} & l_{1} & l_{3} \\
m_{2} & m_{1} & m_{3}
\end{array}\right), \\
& \left(\begin{array}{ccc}
l & l & 0 \\
m & -m & 0
\end{array}\right)=\frac{(-1)^{l-m}}{\sqrt{2 l+1}} \\
& m_{1}+m_{2}+m_{3} \neq 0 \Rightarrow\left(\begin{array}{ccc}
l_{1} & l_{2} & l_{3} \\
m_{1} & m_{2} & m_{3}
\end{array}\right)=0, \\
& \sum_{m_{1} m_{2}}\left(2 l_{3}+1\right)\left(\begin{array}{ccc}
l_{1} & l_{2} & l_{3} \\
m_{1} & m_{2} & m_{3}
\end{array}\right)\left(\begin{array}{ccc}
l_{1} & l_{2} & l_{3}^{\prime} \\
m_{1} & m_{2} & m_{3}^{\prime}
\end{array}\right) \\
& =\delta_{l_{3} l_{3}} \delta_{m_{3} m_{3}^{\prime}} \cdot
\end{aligned}
$$

The relation between spherical harmonics and Wigner $3 \mathrm{j}$ symbols is

$$
\begin{aligned}
& \int d \hat{n} Y_{l_{1} m_{1}}(\hat{\mathbf{n}}) Y_{l_{2} m_{2}}(\hat{\mathbf{n}}) Y_{l_{3} m_{3}}(\hat{\mathbf{n}}) \\
& =\sqrt{\frac{\left(2 l_{1}+1\right)\left(2 l_{2}+1\right)\left(2 l_{3}+1\right)}{4 \pi}}\left(\begin{array}{ccc}
l_{1} & l_{2} & l_{3} \\
m_{1} & m_{2} & m_{3}
\end{array}\right) \\
& \quad \times\left(\begin{array}{ccc}
l_{1} & l_{2} & l_{3} \\
0 & 0 & 0
\end{array}\right)
\end{aligned}
$$

\section{APPENDIX B: ROTATION VS. WEAK LENSING}

The effects of rotation and weak lensing [21,24] on polarization are orthogonal and can thus be distinguished geometrically with a full-sky map. For example, if we start off with a pure $E$ mode at the surface of last scatter, rotation induces a $B$ mode, given by Eq. (6), where the only nonzero terms are those that satisfy $L+l+l_{2}=$ even. However, if we analyze the effect of weak lensing (see Ref. [24]), a pure $E$ mode polarization tensor changes by

$$
\delta P_{a b}=\left(\nabla_{c} \varphi\right)\left(\nabla^{c} P_{a b}\right),
$$

where $\varphi$ is the projection of the gravitational potential along the line of sight. Thus, the $B$ mode induced by weak lensing is 


$$
\begin{aligned}
\delta B_{l m}= & \int d \hat{n} \delta P_{a b}(\hat{n}) Y_{(l m)}^{B * a b}(\hat{n}) \\
= & 2 \sum_{L M} \sum_{l_{2} m_{2}} \varphi_{L M} E_{l_{2} m_{2}} \int d \hat{n} Y_{(l m)}^{B *, a b}\left(\nabla_{c} Y_{(L M)}\right) \\
& \times\left(\nabla^{c} Y_{\left(l_{2} m_{2}\right) a b}^{E}\right) .
\end{aligned}
$$

The parity of the spherical-harmonic $Y_{L M}$ is $(-1)^{L}$. The parity of the $E$-mode term in the integral is $(-1)^{l_{2}}$, and the parity of the $B$-mode term is $(-1)^{l+1}$. The parity of the integrand in Eq. (B2) is then $(-1)^{L+l+l_{2}+1}$. Therefore, the integral is nonvanishing only for terms that satisfy $L+l+$ $l_{2}=$ odd.

We conclude that the rotation induces $B$ modes that satisfy $L+l+l_{2}=$ even, while for weak lensing we have $L+l+l_{2}=$ odd [see Eqs. (6) and (20)]. Thus, the two effects can be entirely separated.

Strictly speaking, this orthogonality between lensing and rotation occurs only at linear order in the rotation and lensing amplitudes in the limit that lensing and rotation are both small. If a lensed field is then rotated, and/or if a rotated field is then lensed, then the orthogonality will break down. However, this will occur only with an amplitude that is proportional to the product of the lensing and rotation amplitudes. We have here implicitly assumed this to be small and leave the full treatment of this higher-order effect for future work.

\section{APPENDIX C: UNIFORM ROTATION CROSS-CHECK}

We can perform a cross-check of our formulas for variances of the rotation, by analyzing only the $L=0$ term, where all the representation-theory coefficients can be readily evaluated. We do so for the EB case. From Eqs. (49) and (50) (for $l=l^{\prime}$ ), after evaluating the coefficients for $L=0$, we get the variance of the uniformrotation estimator to be

$$
\left(\sigma_{00}\right)^{-2}=\frac{1}{\pi} \sum_{l} \frac{\left[C_{l}^{E E}\left(W_{l}\right)^{2}\right]^{2}(2 l+1)}{C_{l}^{E E, \text { map }} C_{l}^{B B, \text { map }}} .
$$

We can see that this is indeed the right expression for uniform rotation, by noting that the $B$ mode induced by small rotation, from a pure $E$ mode, is given by Eq. (3). The induced EB power spectrum in that case is

$$
C_{l}^{E B}=2 \alpha C_{l}^{E E},
$$

and estimators for the rotation can be expressed for each $\mathrm{lm}$ pair as

$$
\hat{\alpha}=\frac{E_{l m}^{\mathrm{map}} B_{l m}^{\mathrm{map}}}{2 C_{l}^{E E} W_{l}^{2}} .
$$

The variance of rotation is then calculated from all $l \mathrm{~m}$ pairs as
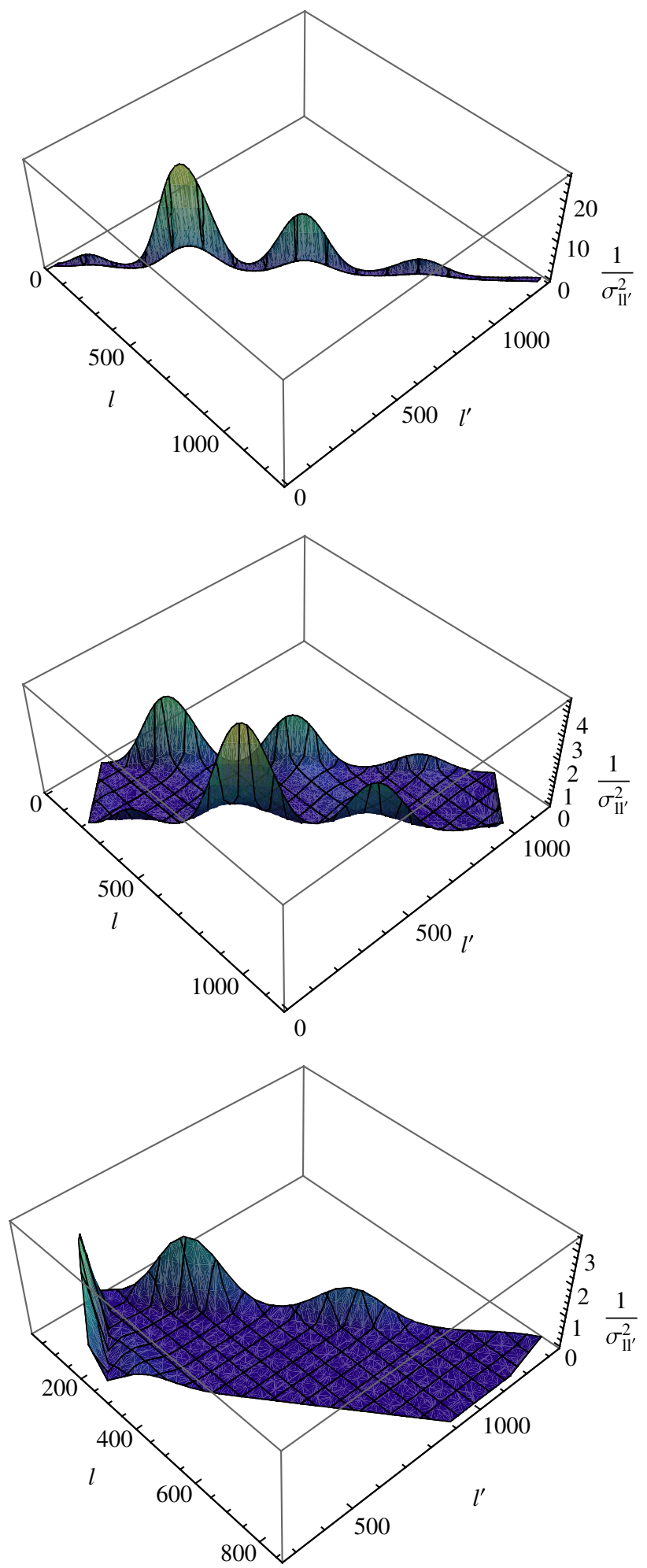

FIG. 6 (color online). The summands in Eq. (47) are plotted versus $l$ and $l^{\prime}$. Plots for three different rotation multipole coefficients are shown: $L=20$ (top panel), $L=200$ (middle panel), and $L=500$ (bottom panel). The plots suggest that the major contribution to any given rotation multipole coefficient comes from the $l l^{\prime}$ estimators that correspond to the strongest peaks in the EE power spectrum above the resolution limit of the instrument. 


$$
\sigma^{-2}=\sum_{l=0}^{\infty} \sum_{m=-l}^{l} \frac{1}{\left\langle(\hat{\alpha})^{2}\right\rangle}=\sum_{l}(2 l+1) \frac{4\left[C_{l}^{E E}\left(W_{l}\right)^{2}\right]^{2}}{C_{l}^{E E \text {,map }} C_{l}^{B B, \text { map }}},
$$

where the factor of $(2 l+1)$ comes from the sum over $m$, because the terms in the sum have effectively only index $l$. For $L=0$, Eq. (C4) reduces to Eq. (C1), once the factor of $\sqrt{4 \pi}$ by which $\alpha$ and $\alpha_{00}$ differ is taken into account.

\section{APPENDIX D: CONTRIBUTIONS OF MULTIPOLE PAIRS TO ROTATION ESTIMATOR}

In Fig. 6, we show the summand in Eq. (47), versus $l$ and $l^{\prime}$, for $L=20,200$, and 500. These terms are the weights with which each $l l^{\prime}$ pair contributes to the EB-estimator $\hat{\alpha}_{L M}$ for Planck [see Eq. (46)]. Figure 6 therefore illustrates which multipoles in the EE power spectrum are expected to contribute most to the three chosen rotation multipoles. From the Figure, we see that the region allowed by triangle inequalities grows with $L$, and that the peaks along $l^{\prime}$ correspond to the peaks in the EE power spectrum. There is also an overall exponential decay from the window functions, with a characteristic scale of $l^{\prime} \sim 1000$, beyond which the distributions fall to zero.

Overall, the EE multipoles that affect the estimate of the rotation at $L=20$ and 200 seem to be predominantly those that correspond to the strongest peaks in the EE power spectrum, below the resolution limit of $l^{\prime} \sim 1000$ (i.e. $l^{\prime}$ of about 400, 700, and 1000; see Fig. 7). The relative con-

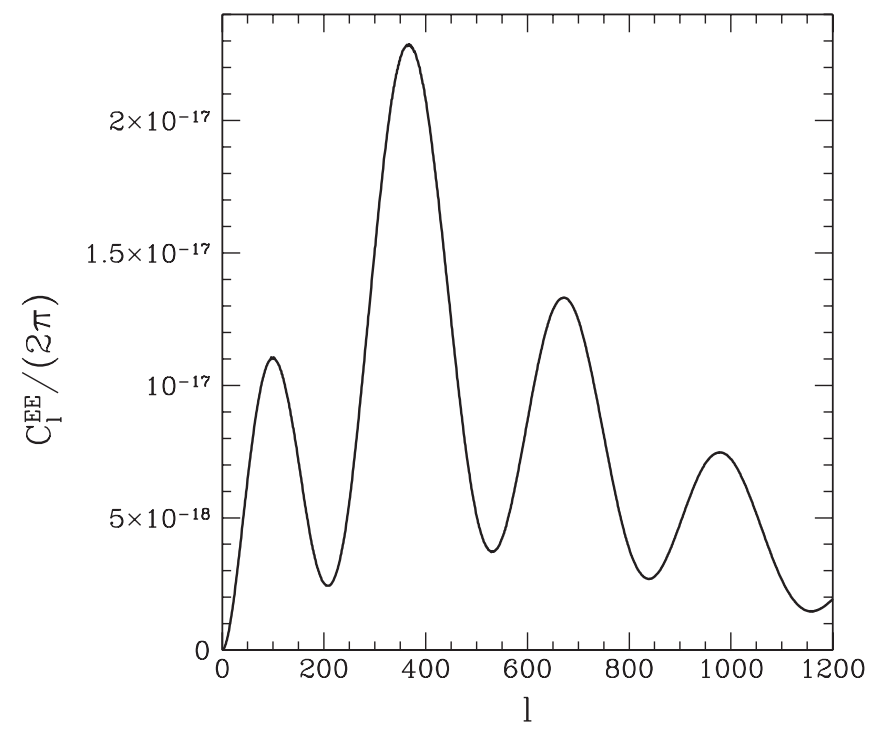

FIG. 7. The EE power spectrum used for numerical estimates in Sec. $\mathrm{V}$ is shown. The main peaks of the power spectrum are visible at $L \sim 150,400,700$, and 700 .

tributions are shown in the top two panels of Fig. 6. The bottom panel of the same Figure shows the $L=500$ case. There, we see that the largest contribution is local, i.e. it comes from nonflat triangles at multipoles below $l^{\prime} \sim 500$, and the secondary contribution comes from the EE powerspectrum peaks at $l^{\prime}$ of about 700 and 1000 (see Fig. 7).
[1] P. de Bernardis et al. (Boomerang Collaboration), Nature (London) 404, 955 (2000); A. D. Miller et al., Astrophys. J. 524, L1 (1999); S. Hanany et al., Astrophys. J. 545, L5 (2000); N. W. Halverson et al., Astrophys. J. 568, 38 (2002); B. S. Mason et al., Astrophys. J. 591, 540 (2003); A. Benoit et al. (Archeops Collaboration), Astron. Astrophys. 399, L25 (2003); J. H. Goldstein et al., Astrophys. J. 599, 773 (2003); D. N. Spergel et al. (WMAP Collaboration), Astrophys. J. Suppl. Ser. 148, 175 (2003); C. L. Reichardt et al., Astrophys. J. 694, 1200 (2009); D. N. Spergel et al. (WMAP Collaboration), Astrophys. J. Suppl. Ser. 170, 377 (2007); J. Dunkley et al. (WMAP Collaboration), Astrophys. J. Suppl. Ser. 180, 306 (2009).

[2] J. Bock et al., arXiv:astro-ph/0604101.

[3] A. H. Guth, Phys. Rev. D 23, 347 (1981); A. Albrecht and P. J. Steinhardt, Phys. Rev. Lett. 48, 1220 (1982); A. D. Linde, Phys. Lett. B 108, 389 (1982).

[4] M. Kamionkowski and A. Kosowsky, Annu. Rev. Nucl. Part. Sci. 49, 77 (1999).

[5] M. Kamionkowski, A. Kosowsky, and A. Stebbins, Phys. Rev. D 55, 7368 (1997); Phys. Rev. Lett. 78, 2058 (1997).
[6] M. Zaldarriaga and U. Seljak, Phys. Rev. D 55, 1830 (1997); U. Seljak and M. Zaldarriaga, Phys. Rev. Lett. 78, 2054 (1997).

[7] M. Kamionkowski and A. Kosowsky, Phys. Rev. D 57, 685 (1998).

[8] S. M. Carroll, Phys. Rev. Lett. 81, 3067 (1998).

[9] A. Lue, L. M. Wang, and M. Kamionkowski, Phys. Rev. Lett. 83, 1506 (1999); N. F. Lepora, arXiv:gr-qc/9812077; K. R. S. Balaji, R. H. Brandenberger, and D. A. Easson, J. Cosmol. Astropart. Phys. 12 (2003) 008.

[10] B. Feng et al., Phys. Rev. Lett. 96, 221302 (2006); T. Kahniashvili, R. Durrer, and Y. Maravin, Phys. Rev. D 78, 123009 (2008); J. Q. Xia et al., Astrophys. J. 679, L61 (2008); , Astron. Astrophys. 483, 715 (2008); E. Y. Wu et al. (QUaD Collaboration), Phys. Rev. Lett. 102, 161302 (2009); L. Pagano et al., arXiv:0905.1651.

[11] E. Komatsu et al. (WMAP Collaboration), Astrophys. J. Suppl. Ser. 180, 330 (2009).

[12] M. Pospelov, A. Ritz, and C. Skordis, arXiv:0808.0673; M. Li and X. Zhang, Phys. Rev. D 78, 103516 (2008).

[13] S. Gardner, Phys. Rev. Lett. 100, 041303 (2008).

[14] M. Kamionkowski, Phys. Rev. Lett. 102, 111302 (2009). 
[15] A. P. S. Yadav, R. Biswas, M. Su, and M. Zaldarriaga, arXiv:0902.4466.

[16] http://www.rssd.esa.int/PLANCK.

[17] J. Bock et al., arXiv:0805.4207.

[18] A.L. Erickcek, S. M. Carroll, and M. Kamionkowski, Phys. Rev. D 78, 083012 (2008); A. L. Erickcek, M. Kamionkowski, and S.M. Carroll, Phys. Rev. D 78, 123520 (2008).

[19] A. R. Pullen and M. Kamionkowski, Phys. Rev. D 76, 103529 (2007).

[20] J. Hoftuft et al., arXiv:0903.1229; F. K. Hansen et al., arXiv:0812.3795; H. K. Eriksen et al., Astrophys. J. 605, 14 (2004); 609, 1198(E) (2004); F. K. Hansen, A. J. Banday, and K. M. Górski, Mon. Not. R. Astron. Soc. 354, 641 (2004); H. K. Eriksen et al., Astrophys. J. 660, L81 (2007).

[21] W. Hu, Phys. Rev. D 62, 043007 (2000).

[22] A. Hajian and T. Souradeep, arXiv:astro-ph/0501001; T.
Souradeep, A. Hajian, and S. Basak, New Astron. Rev. 50, 889 (2006); A. Hajian and T. Souradeep, Phys. Rev. D 74, 123521 (2006); Astrophys. J. 597, L5 (2003); S. Basak, A. Hajian, and T. Souradeep, Phys. Rev. D 74, 021301 (2006).

[23] M. Betoule et al., arXiv:0901.1056.

[24] M. Zaldarriaga and U. Seljak, Phys. Rev. D 58, 023003 (1998); U. Seljak and M. Zaldarriaga, Phys. Rev. Lett. 82, 2636 (1999); M. Zaldarriaga and U. Seljak, Phys. Rev. D 59, 123507 (1999); T. Okamoto and W. Hu, Phys. Rev. D 67, 083002 (2003); W. Hu and T. Okamoto, Astrophys. J. 574, 566 (2002); M. Kesden, A. Cooray, and M. Kamionkowski, Phys. Rev. Lett. 89, 011304 (2002); L. Knox and Y. S. Song, Phys. Rev. Lett. 89, 011303 (2002); M. H. Kesden, A. Cooray, and M. Kamionkowski, Phys. Rev. D 67, 123507 (2003); P. Cabella and M. Kamionkowski, arXiv:astro-ph/0403392; A. Lewis and A. Challinor, Phys. Rep. 429, 1 (2006). 